\title{
Research on Prediction Model of Basic Sintering Characteristics of Mixed Iron Ore and Sinter Quality
}

\author{
Liu Song * \\ College of Metallurgy \& Energy \\ North China University of Science and Technology \\ Tangshan, China \\ E-mail: 358958433@qq.com \\ * Corresponding Author \\ Lu Jianguang \\ School of Materials \& Metallurgy \\ Northeastern University \\ Shenyang, China \\ E-mail: ltbjsk@mail.hgjt.cn
}

\author{
Li Fumin \\ College of Metallurgy \& Energy \\ North China University of Science and Technology \\ Tangshan, China \\ E-mail: lifumin@ncst.edu.cn \\ Lu Qing \\ College of Metallurgy \& Energy \\ North China University of Science and Technology \\ Tangshan, China \\ E-mail: lq@ncst.edu.cn
}

\begin{abstract}
In order to solve the rapid decision of ore blending scheme in iron ore sintering process, the prediction model of the basic sintering characteristics of mixed iron ore and sinter quality has been established by three algorithms including the support vector machines, BP neural network and general regression neural network. The results show, the model based on support vector machine algorithm is better, which can accurately predict the basic sintering characteristics and sinter quality indexes; the accuracy of prediction for assimilation temperature, liquid fluidity and the binding phase strength are $90 \%, 93 \%$ and $91 \%$ respectively, based on the physical and chemical properties of raw material, and the accuracy of prediction for the drum strength and productivity are $89 \%$ and $88 \%$, based on the basic sintering characteristics of mixed iron ore and technical parameters.
\end{abstract}

Keywords-Basic Sintering Characteristics; Sinter Quality; Support Vector Machines; BP Neural Network; GRNN

\section{INTRODUCTION}

The sintering scheme of iron ore is usually based on existing ore program, or micro adjustment with experience and experiments. The method is blinder and cumbersome, difficult to pinpoint the best solution. Domestic and foreign scholars have more research on the optimization of ore blending, but no matter the prediction model of the basic characteristics of the sintering, or the prediction model of the sinter quality is the problem of the low probability of hit rate. To this end, analyze and compare the fit ability and generalization ability of BP neural networks, GRNN (generalized regression neural networks) and SVM (support vector machines) [1-4], Obtaining optimal algorithm. The prediction model of the basic sintering characteristics of mixed iron ore and sinter quality is established, to provide reference for the quick decision of ore blending scheme. 
TABLE I. CHEMICAL COMPOSITION OF THE MIXED IRON ORE

\begin{tabular}{|c|c|c|c|c|c|c|}
\hline Raw material & $\mathbf{T F e} / \%$ & $\mathbf{S i O}_{2} / \%$ & $\mathbf{C a O} / \%$ & $\mathbf{M g O} / \%$ & $\mathbf{A l}_{2} \mathbf{O}_{3} / \%$ & $\mathbf{L O I} / \%$ \\
\hline 1 & 62.05 & 4.17 & 0.15 & 0.21 & 1.50 & 4.95 \\
\hline 2 & 62.28 & 4.43 & 0.22 & 0.30 & 1.45 & 4.48 \\
\hline 3 & 62.50 & 4.68 & 0.29 & 0.38 & 1.41 & 4.02 \\
\hline 4 & 62.73 & 4.94 & 0.36 & 0.47 & 1.36 & 3.56 \\
\hline 5 & 61.53 & 4.42 & 0.23 & 0.29 & 1.44 & 5.35 \\
\hline 6 & 60.95 & 4.83 & 0.16 & 0.21 & 1.47 & 5.98 \\
\hline 7 & 63.45 & 4.38 & 0.36 & 0.47 & 1.31 & 2.98 \\
\hline 8 & 62.87 & 4.80 & 0.29 & 0.39 & 1.34 & 3.60 \\
\hline 9 & 61.38 & 4.53 & 0.30 & 0.38 & 1.36 & 5.39 \\
\hline 10 & 62.34 & 4.51 & 0.36 & 0.47 & 1.30 & 4.21 \\
\hline 11 & 61.69 & 4.81 & 0.16 & 0.22 & 1.40 & 5.20 \\
\hline 12 & 62.65 & 4.79 & 0.22 & 0.30 & 1.34 & 4.01 \\
\hline 13 & 62.33 & 4.23 & 0.36 & 0.46 & 1.26 & 4.35 \\
\hline
\end{tabular}

Using the cut-and-trial, the structure of the three layer network of BP neural network is 6-13-1. The times of model training is 1000 , the goal of it is 0.01 , and the learning rate is 0.1 . The structure of GRNN and SVM are determined by the training sample self-adaption, the model parameters can be obtained by the grid search and crossvalidation. The smoothing parameter of radial basis function of GRNN is 0.42. SVM kernel function is selected by the radial basis kernel function, the penalty function $\mathrm{C}=1.3124$ and the radial basis kernel function $\mathrm{g}=\mathrm{l}$. The fitting results of training set and the prediction results of test set are shown in the Table II.

TABLE II. FITTING EFFECT OF THE TRAINING SET AND PREDICT EFFECT OF THE EXPERIMENT SET

\begin{tabular}{|c|c|c|c|c|c|}
\hline \multirow{2}{*}{ Method } & \multicolumn{2}{|c|}{ Training set } & & \multicolumn{2}{|c|}{ Test set } \\
\cline { 2 - 3 } \cline { 5 - 5 } & $\begin{array}{c}\text { Mean } \\
\text { square } \\
\text { error }\end{array}$ & $\begin{array}{c}\text { Coefficient of } \\
\text { determination }\end{array}$ & & $\begin{array}{c}\text { Mean } \\
\text { square } \\
\text { error }\end{array}$ & $\begin{array}{c}\text { Coefficient of } \\
\text { determination }\end{array}$ \\
\hline BP & 0.0036 & 0.5492 & & 0.0037 & 0.5983 \\
\hline GRNN & 0.0034 & 0.6362 & & 0.0038 & 0.6891 \\
\hline SVM & 0.0019 & 0.7695 & & 0.0027 & 0.7964 \\
\hline
\end{tabular}

It can be seen in Table II that the fitting and generalization of SVM is better than the GRNN and BP neural network. For the fitting of training set and the prediction of test set, the measures are the mean square error and the coefficient of determination. Compared with the other two algorithms, the mean square error of the SVM is minimized and the coefficient of determination of that is closed to 1 , which show that the accuracy of prediction model based on SVM is the highest, and the data of prediction is most accurate.

\section{MODEL ESTABLISHMENT}

\section{A. Confirmation of the Input Parameters}

With studying the effect of properties of raw material on the sintering of iron ore, the factors that could affect the basic sintering characteristics and sinter quality are revealed [11-13]. The input and output relationship of the model is shown in Fig. 1.

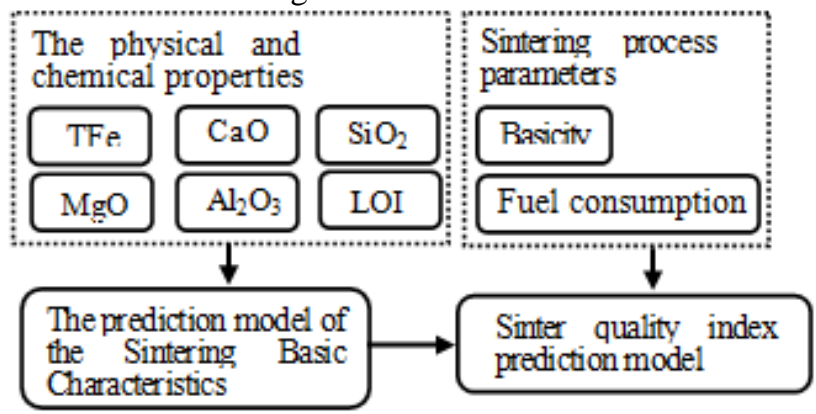

Figure 1. Prediction Model of Basic Sintering Characteristics and Sinter Quality

\section{B. Confirmation of the Kernel}

Using the kernel, the support vector regression algorithm could turn the practical problem into high dimensional feature space by nonlinear mapping and construct the linear regression function in the high dimensional space to achieve the nonlinear regression of the original space. By selecting different kernels, the data can be mapped to different space to form different algorithms. Due to a wide variety of nuclear function, different kernels have different characteristics, therefore, the kinds and parameters of kernel should be selected when solve the practical problem, some kernels are shown:

(1) Polynomial kernel: $K(x, y)=\left(a x^{T} y+c\right)^{d}$

(2) $\mathrm{RBF}$ (Radial basis kernel function):

$$
K(x, y)=\exp \left\{-\frac{\|x-y\|^{2}}{2 \sigma^{2}}\right\}
$$

(3) Sigmoid kernel: $K(x, y)=\tanh \left[a x^{T}+c\right]$

The same training set and test set were compared in order to measure the effect of different types of kernel function on the performance of model, the result is shown in Table III. It can be seen that the order number of polynomial kernel is $a=c=0$ and $d=3$, while the width of the radial basis function is 1.3124, the Sigmoid kernel function is $a=0.1768$ and $c=0$.

TABLE III. EFFECT OF THE TYPE OF KERNEL FUNCTION ON THE RESULTS BOTH OF FITTING AND PREDICTIVE

\begin{tabular}{|c|c|c|c|c|c|}
\hline \multirow{2}{*}{ Kernel } & \multicolumn{2}{|c|}{ Training set } & & \multicolumn{2}{c|}{ Test set } \\
\cline { 2 - 3 } \cline { 5 - 6 } & $\begin{array}{c}\text { Mean } \\
\text { square } \\
\text { error }\end{array}$ & $\begin{array}{c}\text { Coefficient of } \\
\text { determination }\end{array}$ & & $\begin{array}{c}\text { Mean } \\
\text { square } \\
\text { error }\end{array}$ & $\begin{array}{c}\text { Coefficient of } \\
\text { determination }\end{array}$ \\
\hline Polynomial & 0.0030 & 0.7013 & & 0.0023 & 0.5926 \\
\hline RBF & 0.0021 & 0.7653 & & 0.0029 & 0.7489 \\
\hline Sigmoid & 0.0064 & 0.6402 & & 0.0071 & 0.5956 \\
\hline
\end{tabular}

It can be seen in Table III that the fitting and generalization of RBF kernel function are better than polynomial kernel and Sigmoid kernel. However, the parameters of that is less. So the RBF will be the kernel of GRNN model.

\section{Optimization of Model Parameters}

For the optimization of SVM, at present, the most common method is to use grid search combined with cross-validation to optimize parameters. At the same time, 
the research and application of genetic algorithm, particle swarm algorithm and other heuristic algorithms are also gradually increased [14].

The grid search (GS) is to try all kinds of possible parameters matching, cross validation, and take the highest accuracy of cross validation parameters as the best combination of parameters. Genetic algorithm (GA) is a kind of adaptive probability optimization technology, which is based on genetic and evolutionary mechanisms. It has implicit parallelism and powerful global search ability. Particle swarm algorithm (PSO) is an optimization algorithm based on swarm intelligence, searching through the particle in the solution space to follow the best example.

Using the grid search, genetic algorithm and particle swarm algorithm respectively to optimize the model parameters, set $\mathrm{C}$ in the range of $(0 \sim 10), \sigma$ in the range of $(0 \sim 10)$, the maximum evolution generations and population sizes of genetic algorithm and particle swarm are set to 300 and 50 . The result of parameter optimization is shown in Table IV.

TABLE IV. PARAMETER OPTIMIZATION RESULTS

\begin{tabular}{|c|c|c|c|}
\hline Parameter optimization method & GS & GA & PSO \\
\hline $\mathrm{C}$ & 1.3124 & 1.5667 & 1.5106 \\
\hline$\sigma$ & 1.0000 & 1.0435 & 1.0793 \\
\hline
\end{tabular}

The effect of various parameters optimization methods on the results of fitting and prediction is shown in Table V.

TABLE V. EFFECT OF PARAMETER OPTIMIZATION METHOD ON THE RESULTS BOTH OF FITTING AND PREDICTIVE

\begin{tabular}{|c|c|c|c|c|c|}
\hline \multirow{2}{*}{$\begin{array}{c}\text { Parameter } \\
\text { optimization } \\
\text { method }\end{array}$} & \multicolumn{2}{|c|}{ The training set } & & \multicolumn{2}{|c|}{ The experiment set } \\
\cline { 2 - 3 } \cline { 5 - 5 } & $\begin{array}{c}\text { Mean } \\
\text { square } \\
\text { error }\end{array}$ & $\begin{array}{c}\text { Coefficient of } \\
\text { determination }\end{array}$ & & $\begin{array}{c}\text { Mean } \\
\text { square } \\
\text { error }\end{array}$ & $\begin{array}{c}\text { Coefficient of } \\
\text { determination }\end{array}$ \\
\hline GS & 0.0020 & 0.7695 & 0.0023 & 0.7721 \\
\hline GA & 0.0017 & 0.7752 & 0.0026 & 0.7576 \\
\hline PSO & 0.0017 & 0.7798 & 0.0026 & 0.7605 \\
\hline
\end{tabular}

It can be seen in Table $\mathrm{V}$ that after the optimization of genetic algorithm or particle algorithm, the fitting effect of model on the training set is better while the prediction effect on test set is worse than the grid search. Therefore, this study combines the grid search and cross validation to optimize the parameters of the model.

\section{PREDICTION EFFECT}

In the laboratory using TSJ-3 type micro sintering machine, $50 \mathrm{~kg}$ grade sintered cup, the based performance, the drum, and the utilization factor of 13 kinds of mix ore are tested. According to the relationship of input-output, the SVM is used to establish the assimilation temperature prediction model, liquid fluidity prediction model, binder phase strength prediction model, drum strength prediction model and productivity prediction model. The data of prediction is compared with that of experiment, the prediction results of the models are shown in Fig. 2 to Fig.6, the accuracy rate is shown in Table VI.

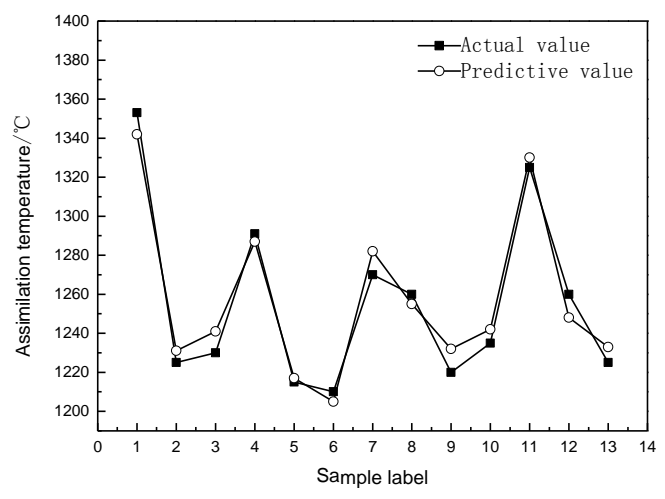

Figure 2. Predicted and Measured Values of Assimilation Temperature

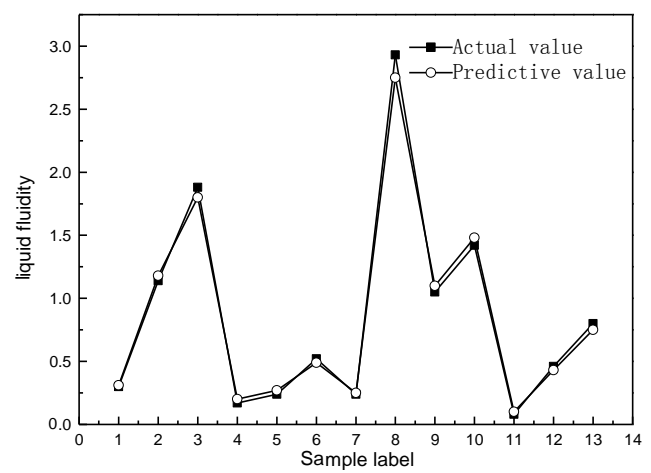

Figure 3. Predicted and Measured Values of Liquid Fluidity

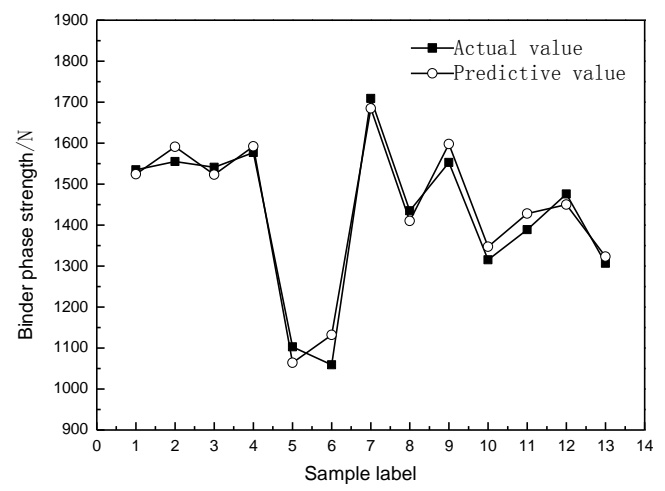

Figure 4. Predicted and Measured Values of Bonding Phase Strength 


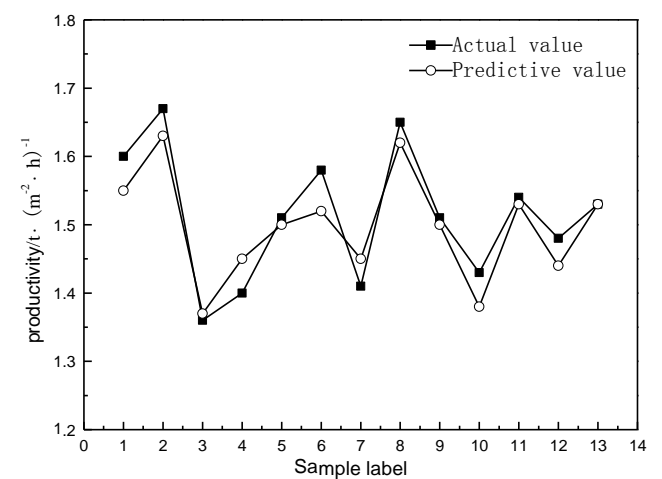

Figure 5. Predicted and Measured Values of Productivity

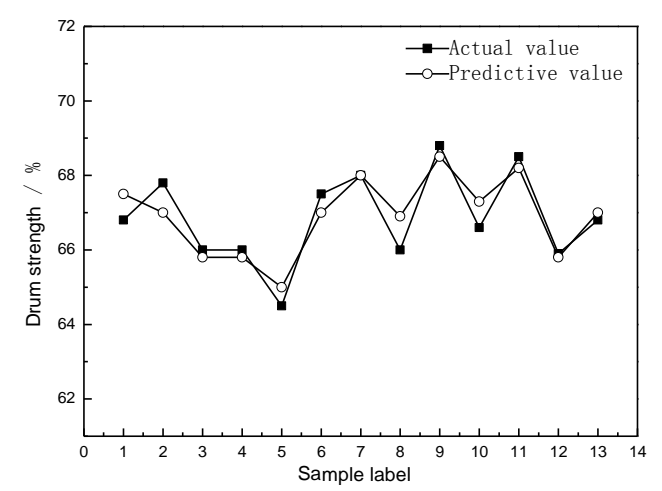

Figure 6. Predicted and Measured Values of Drum Strength

TABLE VI. HIT RATE OF INDEXES BY THE PREDICTION MODEL

\begin{tabular}{|c|c|c|c|c|c|}
\hline \multirow[b]{2}{*}{$\begin{array}{c}\text { Prediction } \\
\text { model }\end{array}$} & \multicolumn{3}{|c|}{ Mixed iron ore powder } & \multicolumn{2}{|c|}{ Sinter } \\
\hline & $\begin{array}{l}\text { Assimilation } \\
\text { temperature }\end{array}$ & $\begin{array}{l}\text { Liquid } \\
\text { fluidity }\end{array}$ & $\begin{array}{c}\text { Binder } \\
\text { phase } \\
\text { strength }\end{array}$ & \begin{tabular}{|} 
Drum \\
strength
\end{tabular} & Productivity \\
\hline $\begin{array}{l}\text { Relative } \\
\text { error } / \%\end{array}$ & \pm 0.6 & \pm 0.6 & \pm 0.8 & \pm 0.8 & \pm 0.8 \\
\hline Hit rate/\% & 90 & 93 & 91 & 89 & 88 \\
\hline
\end{tabular}

As shown in Table VI, the relative error of assimilation temperature, liquid fluidity and binder phase strength is determined by the control precision of micro sintering experiments. The relative error of drum strength and productivity is according to the sintering site requirements. The forecasting results show that the model has good nonlinear fitting ability and generalization ability, and can accurately predict its basic sintering characteristics and sinter quality indicators, so as to realize the optimization of ore blending scheme.

\section{CONCLUSIONS}

1) Compared with the BP neural network, generalized regression neural network and support vector machine fitting and generalization ability, determine to set up based characteristics and sinter quality prediction model based on the support vector machine.

2) SVM kernel function is selected RBF kernel, optimization of the model parameters use the method which grid search and cross-validation are combined. The model has good adaptability, fitting effect and search effect is better.

3) The model can accurately predict the basic sintering characteristics of mixed iron ores and sinter quality indicators, assimilation temperature, liquid fluidity, binder phase strength, drum strength and productivity of the prediction hit rate is respectively $90 \%, 93 \%, 91 \%, 89 \%$ and $88 \%$. Fast ore blending and rapid decision of ore blending plan can be achieved with the model established.

\section{ACKNOWLEDGMENT}

The authors wish to express their acknowledgement to the Natural Science Foundation of Hebei Province - Iron and Steel Co Fund (grant No. E2012209014) for the financial support.

\section{REFERENCES}

[1] Y. H. Chang, Z. Zeng, J. Q. Zhang, Bloom Quality Online Forecasting Model Based on BP Neural Network. Iron and steel, 2011, 46 (5): 33-37.

[2] W N Vapnik, The nature of statistical learning theory. Zhang Xuegong, translation. Beijing: Tsinghua University press, 2000.

[3] L.Q. Meng, M.J. Lei, J.X. Wang, Temperature prediction of plate rolling based on GRNN network. Journal of iron and steel research, 2009, 21 (8): 53-58.

[4] S. F. Ding, B. J. Qi, H. Y. Tan, The theory and algorithm of support vector machines. Journal of University of Electronic Science and technology review, 2011, 40 (1): 2-10.

[5] X. L. Yuan, X. H. Fan, X. Wan, The software design and development of optimizing iron ores matching model. Journal of Central South University (NATURAL SCIENCE EDITION), 2009, 40 (6): $1476-1481$.

[6] C. Zhao, K. Liu, D. S. Li, freight volume prediction based on generalized regression neural network. Journal of the China Railway, 2004, 26 (1): 12-15.

[7] H. Zhou, L. G. Zheng, J. R. Fan, Application of general regression neural network in prediction of coal ash fusion point. Journal of Zhejiang University (Engineering Science Edition), 2004, 38 (11): 1479-1482.

[8] L. M. Li, G. R. Wen, S. C. Wang, Based on genetic algorithm support vector machine regression parameter selection method. Computer engineering and Applications, 2008, 44 (7): 23-26.

[9] S. Q. Xie, F. M. Shen, X. N. Qiu, Face recognition method based on support vector machine. Computer Engineering, 2009, 35 (16): 186-188.

[10] Y. L. Zhang, Network traffic prediction based on genetic algorithm optimization support vector machine. Computer Science, 2008, 35 (5): 177-179.

[11] F. Y. Yi, F. M. Li, K. Sun, The relationship between mixed sintering properties and chemical composition. Sintered pellets, 2013, 38 (2): 1-5.

[12] D. H. Liu, Q. Lv, Y. Q. Sun, The basic characteristics of iron ore influence on sinter properties. Journal of iron and steel research, 2013, 25 (11): 29-34.

[13] Z. Wang, J. L. Zhang, H. B. Zuo, Study on sintering burdening optimization based on high temperature properties of iron ore powder. Sintered pellets, 2013, 38 (3): 1-4.

[14] W. J. Wang, C.S. Men, Support vector machine modeling and application. Beijing: Science Press, 2014. 